\title{
On Impact of Weather on Human Mobility in Cities
}

\author{
Jun Pang, Polina Zablotskaia, and Yang Zhang \\ Computer Science and Communications, University of Luxembourg, Luxembourg
}

\begin{abstract}
Although researchers have demonstrated that human mobility is constrained by space, time and social relations, one important factor, namely weather, has been often ignored in the literature. Not only influences what people wear everyday, weather also has a major impact on their mobility. In this paper, we conduct the first large-scale analysis of weather's impact on human mobility in cities. Focusing on a number of major cities, we construct a human mobility dataset from the social network Instagram. We discover that in general nice weather (e.g., moderate temperature and high pressure) has a positive impact on human mobility. Through analyzing mobility at locations of different categories, we further discover that human mobility is less influenced by weather at certain categories such as residences than others including stores and entertainment places.
\end{abstract}

\section{Introduction}

Urbanization is a massive process happening in this century. Every year, more and more people are moving to cities. According to a $\mathrm{UN}$ report ${ }^{1}$, by 2050 more than 6 billion people will live in cities. Although living in cities brings a lot of convenience to people, it also causes major problems, such as air pollution and traffic congestion. While much effort has been taken to tackle these problems, one fundamental challenge is to fully understand how people move, i.e., human mobility in cities.

Human mobility has attracted the research community a considerable amount of interest during the past decade. Researchers have demonstrated that human mobility is constrained by space, time [1] and social networks [2]. On the other hand, another important factor, i.e., weather, receives much less attention and is often ignored. Weather as a natural phenomenon influences our mobility in many ways. When people check weather reports, they not only decide what to wear, but also where to visit. For instance, few people are willing to walk in a park on a cloudy winter afternoon.

Understanding the relationship between mobility and weather can result in positive benefits for multiple stakeholders: for example, city governors can design specific plans for different weather conditions to control traffic flow; shop owners can provide suitable benefits to attract customers; city residents can choose to visit less crowded places on weekends. In the current work, we conduct the first large-scale analysis of weather's impact on human mobility in cities. Our contributions can be summarized as follows:

- We construct a mobility dataset under different weather parameters for 13 major cities across the world (Section 2). We gather more than 10 millions of users' location records, namely check-ins, from Instagram and weather data from Forecast.io.

\footnotetext{
${ }^{1}$ http://bit. ly/1N3gAH 6
} 


\begin{tabular}{|c|r|r|r||c|r|r|r|}
\hline city & \#check-ins & \#users & \#locations & city & \#check-ins & \#users & \#locations \\
\hline New York & $2,728,705$ & 788,980 & 30,644 & Washington DC & 542,822 & 185,687 & 10,601 \\
\hline Los Angeles & $2,011,106$ & 607,380 & 27,716 & San Francisco & 635,842 & 225,438 & 9,620 \\
\hline Tokyo & 891,029 & 300,111 & 26,586 & Chicago & 725,223 & 233,844 & 12407 \\
\hline London & $1,441,658$ & 516,640 & 15,571 & Rome & 232,305 & 102,022 & 6,267 \\
\hline Paris & 633,868 & 253,516 & 11,112 & Milan & 296,353 & 122,481 & 5,917 \\
\hline Boston & 465,615 & 165,166 & 7,619 & Barcelona & 245,298 & 113,997 & 5,457 \\
\hline Hongkong & 191,899 & 87,413 & 4,203 & & & & \\
\hline \multicolumn{7}{|c|}{ Table 1: Dataset summary. } \\
\hline
\end{tabular}

Table 1: Dataset summary.

- We analyze the relationship between users' general mobility behaviors and different weather parameters such as temperature and humidity (Section 3 ). We quantify users' mobility through average check-in volumes, average movement volumes and average movement distances. Our discoveries, for example, include both low and high temperature have negative effects on mobility; high pressure on the other hand positively affects mobility. Interestingly, we also discover that humidity affects mobility negatively in coastal cities while positively in inland cities.

- We take one step further to analyze users' mobility at different location categories under different weather parameters (Section 4). We discover that users' average check-in volumes at locations of certain categories, such as store, entertainment and professional places, are more correlated with weather than others such as residence places. Moreover, people' movement patterns among location categories are less diverse under a uncomfortable weather condition than a comfortable one.

\section{Dataset Construction}

Check-in data. We collect the geo-tagged photos, i.e., check-ins, in 13 major cities worldwide from Instagram by using its public API from August 1st, 2015 until December 15th, 2015. We first resort to Foursquare to extract all location ids within each city we are interested in, meanwhile collect each location's category information. Then for each Foursquare's location id, we query Instagram's API to get its corresponding location id in Instagram. After obtaining Instagram's location ids, we query each location's recent check-ins several times a day. In the end, more than 10M check-ins have been collected. Table 1 summarizes the dataset. As Foursquare organizes location categories into a tree structure, we take its first level categories including entertainment, food, bar, outdoor, professional, residence, store and transportation to label each location.

Weather. We exploit Forecast.io's API to extract weather data. Forecast.io is a weather application started in 2013, it gathers the data from multiple sources such as NOAA and Met Office, and provides users with the aggregated results. Forecast.io's API provides daily weather data covering temperature $\left({ }^{\circ} \mathrm{C}\right)$, humidity (relative humidity), wind speed (miles per hour), pressure (millibar). In addition, as people normally do not feel the difference when the temperature varies one or two degrees, we bucket temperature into bins of $3^{\circ} \mathrm{C}$ starting from $0^{\circ} \mathrm{C}\left(-2^{\circ} \mathrm{C}-0^{\circ} \mathrm{C}\right)$ to $30^{\circ} \mathrm{C}\left(28^{\circ} \mathrm{C}-30^{\circ} \mathrm{C}\right)$.

\section{Weather and Mobility}

For weather, four parameters are considered including temperature, pressure, wind speed and humidity. For mobility, we focus on two aspects. The first one is the average num- 

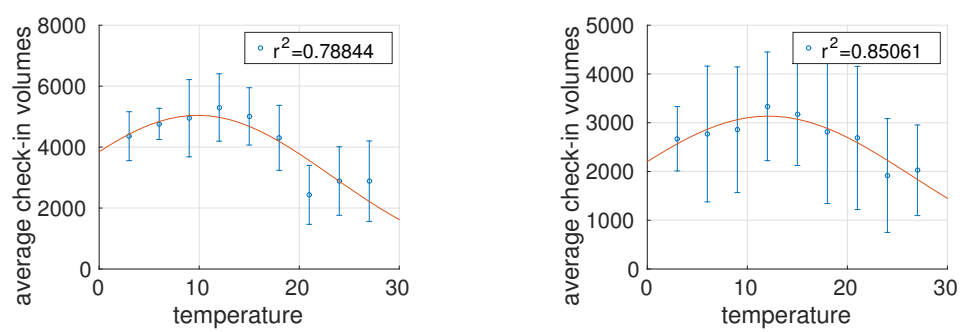

Fig. 1: Temperature vs. average check-in volumes in Paris (left) and Boston (right).

ber of check-ins, namely average check-in volumes, under each value of each weather parameter. The second one is related to users' movements, including the average number of movements, namely average movement volumes and average movement distances $(\mathrm{km})$. Here, we consider a user checking in at two locations within a certain time threshold $\tau$ as one movement. In this paper, we choose $\tau$ to be 3 hours which we believe is a reasonable transition time for a user. The same formulation has been used in [3] as well. Note that our mobility quantification is at a general level instead for each individual user, i.e., we calculate the mean of users' total number of check-ins, number of movements and movement distances under each value of each weather parameter.

Temperature. Figure 1 depicts the average check-in volumes under different temperature (bucketed by $3^{\circ} \mathrm{C}$ ) in Paris and Boston. We observe that people check in more often under a moderate temperature than low $\left(\leq 6^{\circ} \mathrm{C}\right)$ and high $\left(\geq 24^{\circ} \mathrm{C}\right)$ temperature in both cities. We further fit the data into a Gaussian function defined as $a v g_{-} c i(t)=a \cdot \exp \left(-\left(\frac{t-b}{c}\right)^{2}\right)$. In the formula, avg_ci $(t)$ is the average check-in volume at temperature $t$ (bucketed by $3^{\circ} \mathrm{C}$ ), $a, b$ and $c$ are the parameters of the function: $a$ represents the height of the curve peak, $b$ marks the center of the curve and $c$ controls the width. As shown in Figure 1, a high coefficient of determination, i.e., $r^{2}$, is obtained for the fitting, meaning that a Gaussian curve captures the relation between temperature and average check-in volumes. Table 2 lists $r^{2}$ together with parameters $b$ and $c$ for all the cities. We make two interesting observations. First, data for most cities fit the Gaussian function well, except for Los Angeles and London with relatively weak results. This indicates that there exists a universal pattern of temperature's impact on human mobility. Second, the central point of the Gaussian function, i.e., $b$, varies across the cities. Cities located in hot regions such as Hongkong and Los Angeles have higher values for $b$, as people living there are used to hot weather, compared to cities located in cold regions, e.g., London.

For the second aspect of mobility, i.e., average movement volumes and average movement distances, as an example we plot the average movement volumes in Rome and the average movement distances in Washington DC as a function of temperature in Figure 2, respectively. Consistently, we see - similar to average check-in volumes both average movement volumes and distances fit Gaussian functions well.

From the above analysis, we first conclude that human mobility is more adapted to moderate temperature than both low and high temperature.

Pressure. High pressure is a whirling mass of cool and dry air which generally brings good weather, while low pressure is normally associated with bad weather such as cloud, rain and wind. We expect that pressure has positive effects on users' mobility. 


\begin{tabular}{|c|r|r|r||c|r|r|r|}
\hline City & $r^{2}$ & $b$ & $c$ & City & $r^{2}$ & $b$ & $c$ \\
\hline \hline New York & 0.90 & 12.76 & 25.72 & Washington DC & 0.84 & 14.45 & 19.21 \\
\hline Los Angeles & 0.36 & 17.97 & 17.78 & San Francisco & 0.86 & 12.52 & 17.21 \\
\hline Tokyo & 0.67 & 13.77 & 22.92 & Chicago & 0.45 & 13.64 & 23.62 \\
\hline London & 0.24 & 10.97 & 18.92 & Rome & 0.85 & 14.71 & 15.78 \\
\hline Paris & 0.78 & 9.83 & 14.39 & Milan & 0.91 & 10.52 & 15.36 \\
\hline Boston & 0.85 & 12.10 & 20.37 & Barcelona & 0.64 & 16.82 & 21.11 \\
\hline Hongkong & 0.77 & 22.22 & 21.14 & & & & \\
\hline
\end{tabular}

Table 2: $r^{2}$ between temperature and average check-in volumes.
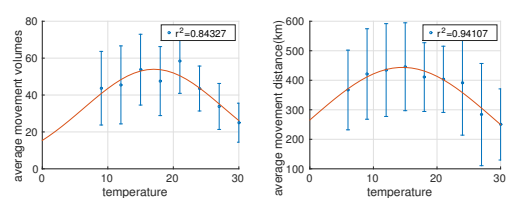

Fig. 2: Temperature vs. average movement volumes in Rome (left) and average movement distances in Washington DC (right).

\begin{tabular}{|c|r|r|r||c|r|r|r|}
\hline City & $r 1$ & $r 2$ & $r 3$ & City & $r 1$ & $r 2$ & $r 3$ \\
\hline \hline New York & 0.23 & 0.60 & 0.68 & Washington DC & 0.74 & 0.69 & 0.66 \\
\hline Los Angeles & 0.16 & 0.21 & 0.12 & San Francisco & 0.31 & -0.20 & -0.22 \\
\hline Tokyo & 0.81 & 0.64 & 0.55 & Chicago & 0.30 & 0.35 & 0.39 \\
\hline London & 0.63 & 0.60 & 0.63 & Rome & 0.57 & 0.09 & 0.05 \\
\hline Paris & 0.54 & 0.48 & 0.47 & Milan & 0.48 & 0.18 & 0.18 \\
\hline Boston & -0.05 & 0.56 & 0.59 & Barcelona & -0.17 & -0.43 & -0.53 \\
\hline Hongkong & 0.25 & 0.18 & 0.18 & & & & \\
\hline
\end{tabular}

Table 3: $r$ between pressure and mobility $(r 1$ : average check-in volumes, $r 2$ : average movement volumes, $r 3$ : average movement distances).
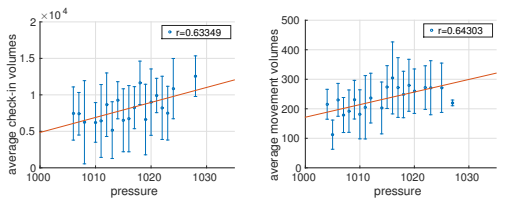

Fig. 3: Pressure vs. check-in volumes in London (left) and movement volumes in Tokyo (right).

Table 3 lists three correlation coefficients $(r)$ between pressure and mobility, pressure indeed positively affects users' mobility in most of the cities. Especially for Tokyo and London, we observe strong correlations (see Figure 3). On the other hand, Barcelona is the only city with three negative correlation coefficients, indicating that pressure has negative effects on human mobility in Barcelona. In addition, most of the cities show consistency between average check-in volumes $(r 1)$ and movements $(r 2, r 3)$, except for San Francisco and Boston. People in San Francisco have more check-ins but less and shorter movements on high pressure days. In Boston, high pressure does not affect average check-in volumes much, instead it leads to more and longer movements.

Wind speed. Wind speed is an important aspect of weather. Through analysis, we discover that average check-in volumes in most cities receives negative wind speed effects (Table 4). Figure 4 (left) presents the result in Barcelona as an example.

The relation between wind speed and movements, on the other hand, is more complicated. For some cities including Los Angeles (see Figure 4 (right)), Tokyo, Paris, Washington DC and Hongkong, wind speed has similar effects on movements as on check-in volumes. On the other hand, in New York, Boston, San Francisco and Barcelona, the (negative) effects of wind on movements become weaker. Moreover, in Chicago, Rome and Milan, there exist positive effects of wind speed on movements. One explanation could be wind negatively affects cycling and walking which results in more car and public transportation usage in these cities. In turn, this leads to the increases in movement volumes and distances. In the end, we observe that in London wind speed has weak effects on average check-in volumes but strong (negative) effects on movements.

Humidity. People normally feel uncomfortable when humidity is low $(\leq 0.3)$ or high $(\geq 0.8)$. Therefore, similar to the case of temperature, we expect the relation between mobility and humidity to follow a Gaussian curve as well. However, analysis results show that humidity (mostly between 0.3 and 0.8 ) and mobility are linearly correlated 


\begin{tabular}{|c|r|r|r||c|r|r|r|}
\hline City & $r 1$ & $r 2$ & $r 3$ & City & $r 1$ & $r 2$ & $r 3$ \\
\hline \hline New York & -0.47 & -0.05 & -0.07 & Washington DC & -0.29 & -0.25 & -0.24 \\
\hline Los Angeles & -0.60 & -0.60 & -0.55 & San Francisco & -0.50 & -0.16 & -0.16 \\
\hline Tokyo & -0.37 & -0.40 & -0.31 & Chicago & -0.26 & 0.26 & 0.30 \\
\hline London & 0.02 & -0.57 & -0.56 & Rome & -0.05 & 0.33 & 0.45 \\
\hline Paris & -0.16 & -0.28 & -0.36 & Milan & -0.31 & 0.24 & 0.21 \\
\hline Boston & -0.40 & -0.03 & -0.01 & Barcelona & -0.56 & -0.18 & -0.07 \\
\hline Hongkong & 0.07 & 0.12 & 0.02 & & & & \\
\hline
\end{tabular}

Table 4: $r$ between wind and mobility ( $r 1$ : average check-in volumes, $r 2$ : average movement volumes, $r 3$ : average movement distances).
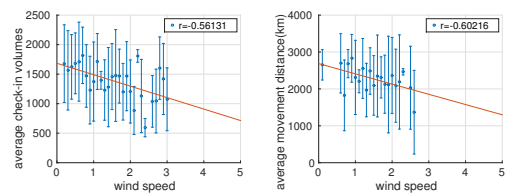

Fig. 4: Wind vs. average check-in volumes in Barcelona (left) and average movement distances in Los Angeles (right).

\begin{tabular}{|c|c|c|c|}
\hline City & $r$ & City & \\
\hline Hongkong & -0.70 & Rome & 0.68 \\
\hline Los Angeles & -0.54 & Paris & 0.61 \\
\hline New York & -0.28 & London & 0.59 \\
\hline Tokyo & -0.19 & Milan & 0.56 \\
\hline San Francisco & -0.14 & Barcelona & 0.50 \\
\hline & & Boston & 0.33 \\
\hline & & Chicago & 0.16 \\
\hline & & Washington DC & 0.12 \\
\hline
\end{tabular}

Table 5: $r$ between humidity and average check-in volumes.

in most of the cities. More interestingly, we observe contradictory linear correlations in different cities. As shown in Figure 5, humidity has positive effects on mobility in Rome while negative effects in Hongkong. Table 5 lists correlation coefficients for both kinds of cities (the results for movements are quite similar and omitted).

Fully understanding the correlation between humidity and mobility involves multiple factors, such as city location, temperature or even culture background, which is out of the scope of the current work. On the other hand, by only studying the dataset, we observe that most of the cities with positive humidity effects are inland cities except for Barcelona and Boston. On the other hand, cities with negative effects are all coastal cities where humidity is normally high. We conjecture that humidity negatively affects human mobility in coastal cities while positively in inland cities.

\section{Weather and Location Category}

In this section we take one step further to analyze weather's influences on mobility at different location categories. We start by analyzing average check-in volumes at each category, then discuss movement patterns among categories.

\subsection{Average check-in volumes}

Temperature. We exploit Gaussian function to model the relation between temperature and average check-in volumes at each location category, assuming that users are more adapted to moderate temperature than both low and high temperature (Section 3 ). Through analysis, we obtain high correlation of determination $\left(r^{2}\right)$ for Gaussian function fitting at entertainment, professional, outdoor and store places. On the other hand, 

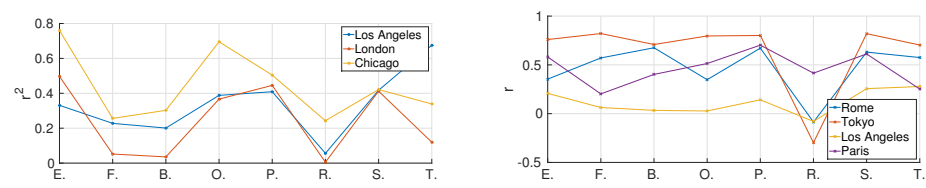

Fig. 6: $r^{2}$ of fitting between average check-in volumes and temperature at location categories (left), $r$ between average check-in volumes and pressure at different location categories (right) (each category is denoted by its first letter).
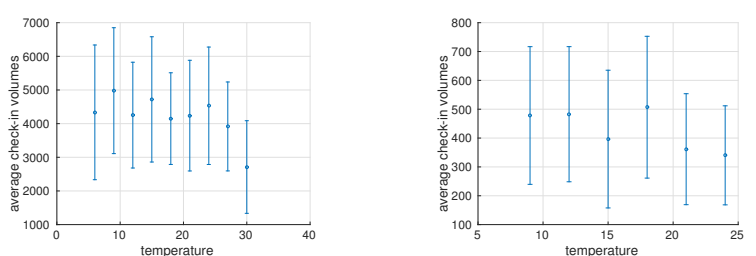

Fig. 7: Average check-in volumes at food places in New York (left) and bar places in San Francisco (right) under different temperature.

residence is the category with the lowest $r^{2}$ values, followed by food and bar. Figure 6 (left) further plots the results in Los Angeles, London and Chicago.

Since Gaussian function cannot capture the correlation between temperature and average check-in volumes at residence, food and bar places, we further examine the data of these categories. Figure 7 depicts the average check-in volumes at food places in New York and bar places in San Francisco as a function of temperature; no clear correlation can be observed. This means whether people going to a bar (a restaurant or a residence) is not strongly dependent on temperature. One reason could be that places of these categories are mostly indoor places, thus not weather-exposed. We conclude that human mobility is more affected by temperature at entertainment, professional, outdoor and store places while it is less affected by temperature at food, bar and residence places.

Pressure. Section 3 states that pressure positively affects users' mobility, this result holds for most of the location categories as well. In addition, in most cities, users' mobility at entertainment, professional and store places receives more positive pressure effects than mobility at other categories. On the other hand, the correlation at residence places is rather weak. For instance, in Figure 6 (right), correlation coefficients $(r)$ at residence places in Tokyo, Rome, Los Angeles and Paris drop quickly when compared to other categories. Meanwhile, there also exist subtle differences among the cities. For instance, users' average check-in volumes at food places have the highest pressure effects in Tokyo while food places have the lowest pressure effects for Paris.

Wind speed. Previously, we have shown that even though wind speed has different effects on movements (average movement volumes and distances) in different cities (Table 4), it still negatively affects average check-in volumes in most cities. However, when conducting analysis at the location category level, similar pattern between wind speed and average check-in volumes cannot be observed. To give an example, we discover that the impact of wind speed at residence places decreases (similar to the cases of temperature and pressure) in some cities, while in other cities the wind's negative 

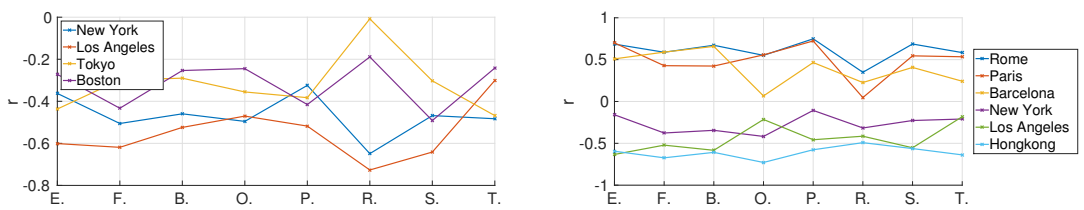

Fig. 8: $r$ between average check-in volumes and wind speed (left) humidity (right) at different location categories.

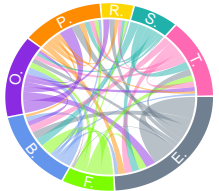

Fig. 9: Movements among categories in London at $12^{\circ} \mathrm{C}$ (left) and $24^{\circ} \mathrm{C}$ (right).
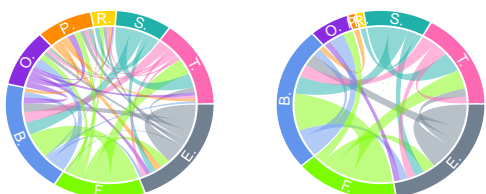

Fig. 10: Movements among categories in Los Angeles at humidity 0.56 (left) and 0.8 (right).

impact even gets stronger. From Figure 8 (left), we see that Tokyo and Boston belong to the former case while New York and Los Angeles represent the latter one.

Humidity. Effects of humidity on users' mobility are positive in some cities while negative in others. For most cities with positive humidity effects, residence is again the category with the lowest correlation coefficients $(r)$ while entertainment, professional and store places have the highest (similar to the results of pressure). For cities with negative humidity effects, we cannot observe a clear pattern. Figure 8 (right) plots the results of a few cites: Rome, Paris and Barcelona for the first case, New York, Los Angles and Hongkong for the second.

\subsection{Movements among location categories}

Since each location is associated with a location category, we further study the weather's impact on movements among different categories. In the current work, we focus on the direct movements between two categories, e.g., from professional places to food places.

Temperature. Among all the 13 cities, we discover that users' movements among location categories under a moderate temperature are more diverse than those under low and high ones. Figure 9 plots two chord diagrams in London under a moderate temperature $\left(12^{\circ} \mathrm{C}\right)$ and a high temperature $\left(24^{\circ} \mathrm{C}\right)$. Each location category is represented by its first letter (capitalized) on the circle, links having the same color as a category are movements starting from that category. Width of each link is proportional to the number of movements. In Figure 9, there exist more links among categories when temperature is $12^{\circ} \mathrm{C}$ than $24^{\circ} \mathrm{C}$. For example, there are many links from entertainment to outdoor and transportation places in the left part of Figure 9, while the number of links decreases on the right part of Figure 9; the most likely destinations for people after checking in at transportation places are bar places when temperature is $12^{\circ} \mathrm{C}$, while they are professional places when temperature is $24^{\circ} \mathrm{C}$.

Humidity. Similar to the case of temperature, users' movements among location categories are more diverse under a comfortable humidity condition than a uncomfortable one. Figure 10 plots the chord diagrams in Los Angeles under two humidity conditions, 
i.e., 0.56 and 0.8 . There are more links in Figure 10 with humidity condition 0.56 than with 0.8 , e.g., many more movements end at outdoor places in the left chord diagram than in the right one. Similar results are obtained for pressure as well.

\section{Related Work}

To the best of our knowledge, the current work is the first large-scale study on weather and human mobility. One close line of work is the study on weather and transportation carried out by the transportation community [4].

Comparing to these studies, our work has the following advantages. 1) Most of the studies conducted by the transportation community focus on weather's impact on people's transportation modes such as public transportation, bicycle or walk. Especially, bicycle usage attracts a lot of attentions (e.g., see $[5,6])$. On the other hand, we focus on users' mobility without any constrains, this makes our analysis more general than theirs. 2) Our dataset is at the global level, i.e., we focus on the mobility of users among 13 cities located in Asia, Europe and North America, while most of the datasets used by the transportation community concentrate on a single city or country. Besides, since our mobility data is from Instagram, the user sample is much bigger than those works whose data is normally collected by conducting surveys.

\section{Conclusion}

We have conducted the first large-scale analysis on the relationship between weather and human mobility in cities. Our discoveries include (1) nice weather, characterized by moderate temperature, high pressure, slow wind speed and suitable humidity, has positive effects on users' mobility; (2) users' mobility at certain location categories, e.g., residence places, is less influenced by weather than mobility at other categories including entertainment, professional and store places.

\section{References}

1. Gonzalez, M., Hidalgo, C., Barabasi, A.L.: Understanding individual human mobility patterns. Nature 453(7196) (2008) 779-782

2. Cho, E., Myers, S.A., Leskovec, J.: Friendship and mobility: user movement in location-based social networks. In: Proc. 17th ACM Conference on Knowledge Discovery and Data Mining (KDD), ACM (2011) 1082-1090

3. Noulas, A., Shaw, B., Lambiotte, R., Mascolo, C.: Topological properties and temporal dynamics of place networks in urban environments. In: Proc. 24th International Conference on World Wide Web (WWW Companion), ACM (2015) 431-441

4. Böcker, L., Dijst, M., Prillwitz, J.: Impact of everyday weather on individual daily travel behaviours in perspective: a literature review. Transport Reviews 33(1) (2013) 71-91

5. Hanson, S., Hanson, P.: Evaluating the Impact of Weather on Bicycle Use. Committee on Bicycle and Bicycle Facilities (1977)

6. Nankervis, M.: The effect of weather and climate on bicycle commuting. Transportation Research Part A: Policy and Practice 33(6) (1999) 417-431 